TRANSACTIONS OF THE

AMERICAN MATHEMATICAL SOCIETY

Volume 365, Number 11, November 2013, Pages 5999-6016

S 0002-9947(2013)05840-0

Article electronically published on August 2, 2013

\title{
SECTIONS OF SURFACE BUNDLES AND LEFSCHETZ FIBRATIONS
}

\author{
R. İNANÇ BAYKUR, MUSTAFA KORKMAZ, AND NAOYUKI MONDEN
}

\begin{abstract}
We investigate the possible self-intersection numbers for sections of surface bundles and Lefschetz fibrations over surfaces. When the fiber genus $g$ and the base genus $h$ are positive, we prove that the adjunction bound $2 h-2$ is the only universal bound on the self-intersection number of a section of any such genus $g$ bundle and fibration. As a side result, in the mapping class group of a surface with boundary, we calculate the precise value of the commutator lengths of all powers of a Dehn twist about a boundary component, concluding that the stable commutator length of such a Dehn twist is $1 / 2$. We furthermore prove that there is no upper bound on the number of critical points of genus $-g$ Lefschetz fibrations over surfaces with positive genera admitting sections of maximal self-intersection, for $g \geq 2$.
\end{abstract}

\section{INTRODUCTION}

Surface bundles over surfaces and, more recently, Lefschetz fibrations, have constituted a rich source of examples of smooth, symplectic, and complex manifolds. The current article explores the existence and the diversity of surface bundles and Lefschetz fibrations which admit sections of maximal possible self-intersection.

Fixing the fiber and the base genera of a surface bundle over a surface, or a Lefschetz fibration, the first question we will tackle is the following: What are the constraints on self-intersection numbers of sections of all such maps? The fundamental obstruction hinges on the fact that the total spaces of these maps are symplectic 4-manifolds and comes from an application of the adjunction inequality for Seiberg-Witten invariants (Proposition 4). Namely, for a given genus- $g$ Lefschetz fibration over a surface $\Sigma_{h}$ of genus $h$ with $g, h \geq 1$, the self-intersection of a section $S$ of this fibration can be at most $2 h-2$. For a surface bundle, the selfintersection number of $S$ is also bounded below by $2-2 h$. (In this article, whenever we talk about a Lefschetz fibration, we will assume the presence of critical points, so as to make a clear distinction.) We refer to these bounds as adjunction bounds and to sections attaining maximal self-intersection numbers allowed by the adjunction bounds as maximal sections.

The natural question to ask, therefore, is whether or not the adjunction bounds are the only universal constraints on the self-intersection number of sections for surface bundles or Lefschetz fibrations with fixed fiber and base genera. We take up this problem in Section 3 for surface bundles over surfaces. We prove that the

Received by the editors October 26, 2011 and, in revised form, February 10, 2012, March 6, 2012, and March 11, 2012.

2010 Mathematics Subject Classification. Primary 57R22, 57R17, 20 F65. 
adjunction bounds are the only global bounds on self-intersections of sections for all $g \geq 2$ and $h \geq 1$. That is, we prove that

Theorem 1. Let $h \geq 1$. For every $g \geq 2$ and $2-2 h \leq k \leq 2 h-2$, there exists a $\Sigma_{g}$-bundle over $\Sigma_{h}$, admitting a section of self-intersection $k$ and, in particular, a maximal section.

We, moreover, construct $\Sigma_{g}$-bundles over $\Sigma_{h}$ which admit disjoint sections that attain all the possible self-intersection numbers between $2-2 h$ and $2 h-2$ for any $g \geq 8 h-8$ and $h \geq 1$ (Theorem [18). These constructions make use of the mapping class group relations we present in the same section.

For the remaining values of $g$ and $h$, we have a complete treatment, which demonstrates a contrast with our results mentioned above. When $g$ or $h$ is zero, the conclusions are classical: For $g=0$, we have ruled surfaces, that is, $S^{2}$-bundles over $\Sigma_{h}$, which can admit sections with arbitrary self-intersections, and for $g \geq 1$ and $h=0$, there is indeed a unique $\Sigma_{g}$-bundle over $S^{2}$ given by projection onto the second factor of $\Sigma_{g} \times S^{2}$. On the other hand, in the case of $g=1$ and $h \geq 1$, we observe that another extreme situation arises: The sections of these elliptic surface bundles always have self-intersection zero (Proposition 20), even though the adjunction bounds hand us a larger range from $2-2 h$ to $2 h-2$ for $h \geq 1$.

A side result of particular interest is given in Theorem 15, where we compute the commutator length of powers of the Dehn twist about a boundary component and, in turn, the stable commutator length of such a Dehn twist. Namely we show:

Theorem 2. Let $g \geq 2, n \geq 1$ and let $\Sigma$ be a compact connected oriented surface of genus $g$ with boundary. If $\delta$ is one of the boundary components of $\Sigma$, then the commutator length of $t_{\delta}^{n}$ is $\lfloor(|n|+3) / 2\rfloor$ and the stable commutator length of $t_{\delta}$ is $1 / 2$.

What follows is a stable calculation which is in great contrast with a result of Kotschick, who showed that the stable commutator length function vanishes when one considers the stable mapping class group of compact surfaces with one boundary component [14]. It follows from our result that the stable commutator length function does not vanish when one works with the stable mapping class group of compact surfaces with more than one boundary component instead. These results are given in Subsection 3.2. Some computations and estimates on the commutator length and the stable commutator length of a (multi-)twist were obtained in [13, 17, 11, 15, 5].

Lastly, we address the following question: For fixed fiber and base genera, is there an upper bound on the number of singular fibers of a relatively minimal Lefschetz fibration admitting a section of maximal self-intersection? As the maximal selfintersection number for a Lefschetz fibration (again, with critical points) over the 2 -sphere is -1 [20, 23], when the base genus $h=0$ this question is due to Ivan Smith (presented by Denis Auroux in [1]). For fiber genus $g \geq 2$ and base genus $h \geq 1$, we show that the answer to this question is negative by proving the following:

Theorem 3. Let $g \geq 2$ and $h \geq 1$ be fixed integers. For any positive integer $M$, there exists a relatively minimal genus-g Lefschetz fibration over a surface of genus $h$ admitting a maximal section such that the number of critical points is greater than $M$.

These results are collected in Section 4. 


\section{Preliminaries}

2.1. Lefschetz fibrations and monodromy representations. In this article, all manifolds are assumed to be smooth and oriented, and all maps are assumed to be smooth. We denote by $\Sigma_{g, r}^{s}$ a compact oriented surface of genus $g$ with $s$ boundary components and $r$ marked points in the interior. The mapping class group,$\Gamma_{g, r}^{s}$, of $\Sigma_{g, r}^{s}$ is the group of isotopy classes of orientation-preserving selfdiffeomorphisms of $\Sigma_{g, r}^{s}$ fixing $r$ marked points and the points on the boundary. The isotopies of $\Sigma_{g, r}^{s}$ are assumed to fix the marked points and the points on the boundary. For simplicity, we write $\Sigma_{g, r}=\Sigma_{g, r}^{0}, \Sigma_{g}^{s}=\Sigma_{g, 0}^{s}$ and $\Sigma_{g}=\Sigma_{g, 0}^{0}$. We also use the similar simplified notation for the corresponding mapping class groups.

We start by reviewing some basic definitions and properties of Lefschetz fibrations and surface bundles over surfaces.

Let $X$ and $\Sigma$ be compact connected manifolds of dimensions four and two, respectively. A Lefschetz fibration is a map $f: X \rightarrow \Sigma$ such that $f^{-1}(\partial \Sigma)=\partial X$, the set $C=\left\{p_{1}, p_{2}, \ldots, p_{k}\right\}$ of critical points of $f$ lies in the interior of $X$, and around each $p_{i}$ and $f\left(p_{i}\right)$ there are orientation-preserving charts making $f$ conform to the complex model $f\left(z_{1}, z_{2}\right)=z_{1} z_{2}$. The genus $g$ of a regular fiber $F \cong \Sigma_{g}$ of $f$ is called the genus of the fibration. We will assume that the critical points lie in different fibers, called singular fibers, which can be achieved after a small perturbation of any given Lefschetz fibration. When there are no critical points, $f: X \rightarrow \Sigma$ is nothing but a surface bundle over a surface, so $f$ always restricts to a surface bundle over $\Sigma \backslash f(C)$ on $X \backslash f^{-1}(f(C))$ and, in particular, over $\partial \Sigma$ on $\partial X$. Below, whenever we talk about a Lefschetz fibration, we will assume that the critical locus is nonempty, so as to make a clear distinction from surface bundles over surfaces.

A singular fiber is called reducible if the complement of the critical point in the fiber is disconnected and is called irreducible otherwise. Lefschetz singularities locally correspond to 2-handle attachments to $D^{2} \times F$ with framing -1 with respect to the fiber framing, where the attaching circles of these 2-handles are embedded curves in a regular fiber $F$ and are called vanishing cycles. With this in mind, a reducible (resp. irreducible) singular fiber is given by a separating (resp. nonseparating) vanishing cycle on $F$. So a reducible fiber consists of two surfaces of self-intersection -1 intersecting each other at the critical point. If one of these two surfaces is a 2 -sphere, that is, if the vanishing cycle is null-homotopic on $F$, then one gets a new Lefschetz fibration by blowing-down this sphere without altering the rest of the fibration, and vice versa. Therefore, we may consider only relatively minimal Lefschetz fibrations, i.e. fibrations which do not contain any sphere of self-intersection -1 in its fibers.

Lefschetz fibrations can be described combinatorially by means of their monodromy. For a smooth surface bundle $f: E \rightarrow \Sigma$ with fibers diffeomorphic to $F$, the monodromy representation of $f$ is defined to be the map $\Psi: \pi_{1}(\Sigma) \rightarrow \Gamma_{g}$ relative to a fixed identification $\varphi$ of $F$ with the fiber over the base point of $\Sigma$ : For each loop $\gamma: I \rightarrow \Sigma$ the bundle $f_{\gamma}: \gamma^{*}(E) \rightarrow I$ is canonically trivial, inducing a diffeomorphism $f_{\gamma}^{-1}(0) \rightarrow f_{\gamma}^{-1}(1)$ up to isotopy. Using $\varphi$ to identify $f_{\gamma}^{-1}(0)$ and $f_{\gamma}^{-1}(1)$ with $F$, we get the element $\Psi(\gamma) \in \Gamma_{g}$. Changing the identification $\varphi$ changes $\Psi$ by a conjugation with an element of $\Gamma_{g}$. Here the map $\Psi: \pi_{1}(\Sigma) \rightarrow \Gamma_{g}$ is an antihomomorphism rather than a homomorphism, because for the multiplication in the mapping class group we use the functional notation, i.e. for $f_{1}, f_{2} \in \Gamma_{g}$, the product $f_{1} f_{2}$ means that we first apply $f_{2}$ and then $f_{1}$. 
For a relatively minimal, genus $-g$ Lefschetz fibration $f: X \rightarrow \Sigma$ with a regular fiber $F$, we define the monodromy representation (or simply monodromy) to be the monodromy factorization of the fiber bundle $X \backslash f^{-1}(Q) \rightarrow \Sigma \backslash Q$, where $Q=f(C)$ is the set of critical values. For $f: X \rightarrow \Sigma$ as above, the monodromy representation $\Psi: \pi_{1}(\Sigma \backslash Q) \rightarrow \Gamma_{g}$ determines $f$ up to isomorphism, except in the cases of sphere and torus bundles over closed surfaces. This is due to the fact that for $g \geq 2$ the space of self-diffeomorphisms of $F$ isotopic to the identity is contractible.

It turns out that the monodromy of a Lefschetz fibration $f: X \rightarrow D^{2}$ over the disk with a single critical point is a right Dehn twist along the vanishing cycle corresponding to the singular fiber. Therefore, the monodromy of a Lefschetz fibration $f: X \rightarrow \Sigma_{h}$ is given by a factorization of the identity element $1 \in \Gamma_{g}$ as

$$
1=\prod_{i=1}^{n} t_{v_{i}} \prod_{j=1}^{h}\left[\alpha_{j}, \beta_{j}\right],
$$

where $v_{i}$ are the vanishing cycles of the singular fibers and $t_{v_{i}}$ is the right (or positive) Dehn twist about $v_{i}$. This factorization of the identity is called the monodromy factorization. In particular, for $F=\Sigma_{g}$, a product $\prod_{i=1}^{h}\left[a_{i}, b_{i}\right]$ of $h$ commutators in $\Gamma_{g}$ gives a $\Sigma_{g}$-bundle over the surface $\Sigma_{h}^{1}$ of genus $h$ with one boundary component. The mapping classes $a_{i}$ and $b_{i}$ specify the monodromies along a free generating system $\left\langle\alpha_{1}, \beta_{1}, \ldots, \alpha_{h}, \beta_{h}\right\rangle$ of $\pi_{1}\left(\Sigma_{h}^{1}\right)$ such that $\prod_{i=1}^{h}\left[\alpha_{i}, \beta_{i}\right]$ is parallel to the boundary component of $\Sigma_{h}^{1}$. If $\prod_{i=1}^{h}\left[\alpha_{i}, \beta_{i}\right]=1$ in $\Gamma_{g}$, we get a $\Sigma_{g}$-bundle $X \rightarrow \Sigma_{h}$. The bundle is uniquely determined by this factorization of the identity once $g \geq 2$.

Conversely, a product $\prod_{i=1}^{k} t_{i} \in \Gamma_{g}$ with $t_{i}$ right Dehn twists provides a genus $-g$ Lefschetz fibration $X \rightarrow D^{2}$ over the disk with fiber $F \cong \Sigma_{g}$. So if $\prod_{i=1}^{k} t_{i}=1$ in the mapping class group $\Gamma_{g}$, then the fibration closes up to a fibration over the sphere $S^{2}$ and the closed-up manifold is uniquely determined by the word $\prod_{i=1}^{k} t_{i}$ once $g \geq 2$. By combining the above two constructions, a word

$$
w=\prod_{i=1}^{k^{\prime}} t_{i} \prod_{j=1}^{h}\left[\alpha_{i}, \beta_{i}\right]
$$

gives a Lefschetz fibration over $\Sigma_{h} \backslash D^{2}$, and if $w=1$ in $\Gamma_{g}$ we get a Lefschetz fibration $X \rightarrow \Sigma_{h}$.

For a Lefschetz fibration or a surface bundle $f: X \rightarrow \Sigma$, a map $\sigma: \Sigma \rightarrow X$ is called a section if $f \circ \sigma=i d_{\Sigma}$. Suppose that a fibration $f: X \rightarrow \Sigma$ admits a section $\sigma$. Set $S=\sigma(\Sigma) \subset X$. We will also say that $S$ is a section of $f$. This section $S$ provides a lift of the representation $\Psi$ from $\pi_{1}(\Sigma \backslash Q)$ to the mapping class group $\Gamma_{g, 1}$. One can then fix a disk neighborhood of this section preserved under the monodromy and get a lift to $\Gamma_{g}^{1}$.

Conversely, every such representation with a lift determines a fibration with a section: Gluing a disk with a marked point to a surface with one boundary component along the boundary and by extending self-diffeomorphisms of the surface by the identity on the disk, we obtain a surjective homomorphism $p: \Gamma_{g}^{1} \rightarrow \Gamma_{g, 1}$. It is well known that ker $p$ is isomorphic to $\mathbb{Z}$, generated by the right Dehn twist $t_{\delta}$ along a simple closed curve $\delta$ parallel to the boundary. If the factorization

$$
1=\prod_{i} t_{v_{i}} \prod_{j}\left[\alpha_{j}, \beta_{j}\right]
$$


lifts from $\Gamma_{g}$ to $\Gamma_{g, 1}$ as a factorization of 1 in the latter group in a similar form, then the corresponding fibration has a section. Moreover, if we lift this product to $\Gamma_{g}^{1}$ we get

$$
t_{\delta}^{m}=\prod_{i} t_{v_{i}^{\prime}} \prod_{j}\left[\alpha_{j}^{\prime}, \beta_{j}^{\prime}\right]
$$

for some $m$. Here, $t_{v_{i}^{\prime}}$ is a Dehn twist mapped to $t_{v_{i}}$ under $\Gamma_{g}^{1} \rightarrow \Gamma_{g}$. Similarly, $\alpha_{j}^{\prime}$ and $\beta_{j}^{\prime}$ are mapped to $\alpha_{j}$ and $\beta_{j}$, respectively. An elementary observation is that the power $m$ of $t_{\delta}$ in the above factorization in $\Gamma_{g}^{1}$ is the negative of the self-intersection number of the section $S$ that we obtain. (See for instance [20].)

2.2. Background results. There are three basic operations we are going to use to construct new surface bundles and Lefschetz fibrations from given ones:

(1) Let $f: X \rightarrow \Sigma_{h}$ be a Lefschetz fibration with regular fiber $\Sigma_{g}$. If $\lambda$ is an orientation-preserving self-diffeomorphism of $\Sigma_{h}$, then $\lambda \circ f: X \rightarrow \Sigma_{h}$ is also a Lefschetz fibration with regular fiber $\Sigma_{g}$. If $f: X \rightarrow \Sigma_{h}$ is a $\Sigma_{g}$-bundle, then one can take the diffeomorhism $\lambda$ above to be orientation-reversing as well to get a new $\Sigma_{g}$-bundle $\lambda \circ f: X \rightarrow \Sigma_{h}$.

(2) For $i=1,2$, let $f_{i}: X_{i} \rightarrow \Sigma_{h_{i}}$ be two genus-g Lefschetz fibrations. We can then remove a fibered neighborhood of a regular fiber $\Sigma_{g}$ from each fibration and glue the resulting 4-manifolds along their boundaries using a fiber-preserving and orientation-reversing diffeomorphism $\phi$ of $S^{1} \times \Sigma_{g}$ to get a new oriented 4-manifold $X$. The result is a new genus- $g$ Lefschetz fibration $f=f_{1} \#_{\phi} f_{2}: X \rightarrow \Sigma_{h_{1}+h_{2}}$ called the fiber sum of $f_{1}$ and $f_{2}$. Moreover, if $S_{i}$ is a section of $f_{i}$ with self-intersection $k_{i}$ for $i=1,2$, then one can perform this fiber sum operation so that there is a section $S_{1} \# S_{2}$, restricting to $S_{i}$ on each fiber sum component, of the fibration $f=f_{1} \#_{\phi} f_{2}: X \rightarrow \Sigma_{h_{1}+h_{2}}$ with self-intersection $k=k_{1}+k_{2}$. In this case, we say that the pair $(f, S)$ is the fiber sum of $\left(f_{1}, S_{1}\right)$ and $\left(f_{2}, S_{2}\right)$.

(3) Let $f_{i}: X_{i} \rightarrow \Sigma_{h}$ be a genus- $g_{i}$ Lefschetz fibration with the regular fiber $\Sigma_{g_{i}}$ with a self-intersection zero section $S_{i}$ for each $i=1,2$. We can then remove a $D^{2}$ fibered neighborhood of each $S_{i}$ in $X_{i}$ and glue the resulting 4-manifolds along their boundaries using a fiber-preserving orientation-reversing diffeomorphism $\phi$ of $S^{1} \times \Sigma_{h}$. The result is a new Lefschetz fibration $f=f_{1} \#_{\phi} f_{2}: X \rightarrow \Sigma_{h}$ with the regular fiber $\Sigma_{g_{1}+g_{2}}$.

The constructions (2) and (3) above are both instances of the generalized fiber sum construction, although they appear to be "orthogonal" to each other in nature. When the fibers are homologically essential (in particular, when the fiber genus $g \neq 1$ ), these Lefschetz fibrations can be equipped with symplectic forms that make the fibers and any prescribed finite collection of disjoint sections symplectic, allowing us to perform this generalized fiber sum construction symplectically and handing us a new symplectic Lefschetz fibration at the end. It is not hard to express all these operations in terms of factorizations and lifts in appropriate mapping class groups.

The next proposition prescribes the adjunction bounds for self-intersection numbers of sections of surface bundles and of Lefschetz fibrations:

Proposition 4 (Adjunction bounds). Let $f: X \rightarrow \Sigma_{h}$ be a surface bundle or a Lefschetz fibration with regular fiber $\Sigma_{g}$, and $g, h \geq 1$. Suppose that $f$ admits a section $S$. Then the self-intersection of $S$ satisfies $[S]^{2} \leq 2 h-2$. If $f: X \rightarrow \Sigma_{h}$ is a $\Sigma_{g}$ bundle, then this bound improves to $\left|[S]^{2}\right| \leq 2 h-2$. 
Proof. If $f$ is a surface bundle over a surface with positive fiber and base genera, we easily deduce from the homotopy exact sequence of a fibration that the total space $X$ is acyclic and, in particular, minimal. On the other hand, if $f$ is a Lefschetz fibration which is not relatively minimal, then we can always blow-down the exceptional spheres on the fibers and pass to a relatively minimal genus $g$ Lefschetz fibration over a genus $h$ surface. Since a section intersects each fiber positively at one point, it either intersects each one of these exceptional spheres positively once or misses it. We therefore obtain a relatively minimal Lefschetz fibration with a section whose self-intersection number is greater than or equal to the self-intersection number of the original section. Thus, it suffices to prove the proposition for a relatively minimal Lefschetz fibration $f$, which we will assume from now on. Then it follows from 22 that the total space of this fibration is minimal. To sum up: in all cases we can assume that we are working with a minimal $X$.

Since the fibration admits a section, the fibers are homologically essential, allowing us to equip $X$ with a symplectic form using Gompf-Thurston construction. We therefore have a minimal symplectic 4-manifold $X$ in hand. In particular, $b^{+}(X) \geq 1$.

The case $b^{+}(X)>1$. The proof in this case will follow at once from an application of the adjunction inequality for Seiberg-Witten basic classes. As shown by Taubes, the canonical class $K$ of any symplectic form we choose on $X$ is a Seiberg-Witten basic class. Applying the adjunction inequality we get

$$
-\chi(S) \geq[S]^{2}+|K \cdot S|
$$

It follows that $2 h-2 \geq[S]^{2}$, proving the first claim of the proposition in this case.

The case $b^{+}(X)=1$. First observe that if $F \cong \Sigma_{g}$ is a regular fiber, then for any $r>\frac{1}{2}\left|[S]^{2}\right|$ the class $r[F]+[S]$ has a positive square, whereas $r[F]-[S]$ has a negative square. So $b^{-}(X) \geq 1$. Moreover, from the exact sequence

$$
\pi_{1}(F) \rightarrow \pi_{1}(X) \stackrel{f_{*}}{\rightarrow} \pi_{1}\left(\Sigma_{h}\right) \rightarrow 1,
$$

we get $b_{1}(X) \geq b_{1}\left(\Sigma_{h}\right)=2 h$. (See for instance [8].)

Second, we show that $X$ is not ruled. From $b_{1}(X) \geq 2 h$, it is fairly easy to see that $X$ can only be a ruled surface over $\Sigma_{n}$ with $n \geq h$. In this case, from the Euler characteristic calculation $4(g-1)(h-1)+m=4-4 n$, where $m$ is the number of Lefschetz critical points, we get

$$
0 \leq \frac{m}{4}=-g h+g+h-n \leq g(1-h) \leq 0 .
$$

Hence, $m=0$ (and $h=n=1$ ), i.e. $f$ should be a surface bundle over a surface. Since the base and the fiber genera are positive, this in turn implies that $\pi_{2}(X)=0$, so $X$ cannot be ruled.

Since $X$ is a minimal symplectic 4-manifold with $b^{+}(X)=1$ and since it is not (irrational) ruled, as shown by Liu in [16, we have the inequality

$$
0 \leq c_{1}^{2}(X)=2 \mathrm{e}(X)+3 \operatorname{sign}(X),
$$

where $\mathrm{e}(X)$ and $\operatorname{sign}(X)$ are the Euler characteristic and the signature of $X$, respectively. From this equation we derive that

$$
0 \leq 4-4 b_{1}(X)+5 b^{+}(X)-b^{-}(X)=9-4 b_{1}(X)-b^{-}(X) .
$$


Therefore, we get

$$
0 \leq 8 h+b^{-}(X) \leq 4 b_{1}(X)+b^{-}(X) \leq 9,
$$

giving us $b_{1}(X)=2, h=1$ and $b^{-}(X)=1$. In particular, the Euler characteristic of $X$ is $\mathrm{e}(X)=0$. Calculating the Euler characteristic from the handle decomposition of $X$ we have

$$
4(g-1)(h-1)+m=0,
$$

where $m$ is the number of singular fibers of the Lefschetz fibration $f$ (considering the surface bundle as a Lefschetz fibration with no singular fiber). We conclude that $m=0$, that is, we have an honest $\Sigma_{g}$-bundle over the torus $T^{2}$.

Let $[S]^{2}=s$. This hands us a relation $t_{\delta}^{-s}=\left[\alpha^{\prime}, \beta^{\prime}\right]$ in the mapping class group $\Gamma_{g}^{1}$ of a surface $\Sigma_{g}^{1}$ with one boundary component, which is a lift of the monodromy factorization $[\alpha, \beta]=1$ in $\Gamma_{g}$ of the surface bundle $f$. Here $\delta$ is the boundary curve $\Sigma_{g}^{1}$. Gluing a torus with one boundary component to $\Sigma_{g}^{1}$ along $\delta$ gives an embedding $\Sigma_{g}^{1} \hookrightarrow \Sigma_{g+1}$, which in turn induces an injection $\Gamma_{g}^{1} \hookrightarrow \Gamma_{g+1}$. Thus we get the relation $t_{\delta^{\prime}}^{-s}=\left[\alpha^{\prime \prime}, \beta^{\prime \prime}\right]$ in $\Gamma_{g+1}$, where $\delta^{\prime}$ is a homotopically nontrivial separating simple closed curve on $\Sigma_{g+1}$. However, it was shown by Endo and Kotschick in [7] that no nontrivial power of a Dehn twist along a separating simple closed curve is a commutator. Therefore, $[S]^{2}=0$, concluding the proof of the first claim.

If $f$ is an honest $\Sigma_{g}$-bundle over $\Sigma_{h}$ with a section $S$ of self-intersection $k$, then we have

$$
\left[\alpha_{1}, \beta_{1}\right]\left[\alpha_{2}, \beta_{2}\right] \cdots\left[\alpha_{h}, \beta_{h}\right]=t_{\delta}^{-k}
$$

in $\Gamma_{g}^{1}$, where $\delta$ is the boundary component. By inverting this equation we obtain the relation

$$
\left[\beta_{h}, \alpha_{h}\right] \cdots\left[\beta_{2}, \alpha_{2}\right]\left[\beta_{1}, \alpha_{1}\right]=t_{\delta}^{k}
$$

which gives another $\Sigma_{g}$-bundle over $\Sigma_{h}$, namely the reflection of $f$. This bundle has a section of self-intersection $-k$. By the result above $-k \leq 2 h-2$; thus $\left|[S]^{2}\right| \leq 2 h-2$, concluding the proof.

While circulating an earlier version of this article, we found out that such an adjunction bound in the case of surface bundles of fiber genera $g \geq 2$ was independently obtained by Bowden (Proposition 3.2 in [3]), where the author studies multi-sections of surface bundles.

Note that when we have a Lefschetz fibration there is indeed no lower bound on the self-intersection of a section. This can be seen for instance by taking fiber sums with appropriate Lefschetz fibrations over the 2-sphere with negative square sections while patching the sections.

When the base genus is $h=0$, a maximally self-intersecting section for a Lefschetz fibration (again with nonempty critical locus) has self-intersection -1 , as shown in $[20,23$. In light of the above proposition, we make the following definition:

Definition 5. Let $f: X \rightarrow \Sigma_{h}$ be a Lefschetz fibration with the regular fiber $\Sigma_{g}$ and let $S$ be a section of this fibration. Then $S$ is called a maximally self-intersecting section, or a maximal section for short, if $[S]^{2}=2 h-2$ when $h \geq 1$ and $[S]^{2}=-1$ when $h=0$. 
Let us now define:

Definition 6 (Fiber sum indecomposability for pairs). Let $f$ be a surface bundle or a Lefschetz fibration over $\Sigma_{h}$ with the regular fiber $\Sigma_{g}$ and let $S$ be a section of this fibration. If $(f, S)$ can be expressed as the fiber sum of $\left(f_{1}, S_{1}\right)$ and $\left(f_{2}, S_{2}\right)$ for some surface bundles or Lefschetz fibrations $f_{i}: X_{i} \rightarrow \Sigma_{g_{i}}$ with sections $S_{i}$ such that neither one of $\left(f_{i}, S_{i}\right)$ is the trivial $\Sigma_{g}$-bundle over $S^{2}$, then $(f, S)$ is said to be fiber sum decomposable. The pair $(f, S)$ is called fiber sum indecomposable otherwise. Fiber sum indecomposability for a fibration $f$ alone (without any mentioning of a section) is defined similarly.

Proposition 7. Let $g, h \geq 1$. If $f: X \rightarrow \Sigma_{h}$ is a surface bundle or a Lefschetz fibration with the regular fiber $\Sigma_{g}$ admitting a maximal section $S$, then the pair $(f, S)$ is fiber sum indecomposable.

Proof. Let $(f, S)$ be the fiber sum of two genus $-g$ fibrations $\left(f_{1}, S_{1}\right)$ and $\left(f_{2}, S_{2}\right)$ over surfaces of genera $h_{1}$ and $h_{2}$, respectively. Assume that $0<h_{1} \leq h_{2}<h$. We have $\left[S_{1}\right]^{2}+\left[S_{2}\right]^{2}=[S]^{2}=2 h-2$ and $h_{1}+h_{2}=h$. However, by Proposition 4 . $\left[S_{i}\right]^{2} \leq 2 h_{i}-2$ for $i=1,2$, yielding a contradiction. So now assume that $h_{1}=0$ and $h_{2}=h$. Once again by Proposition $4\left[S_{2}\right]^{2} \leq 2 h-2$. It follows that $\left[S_{1}\right]^{2}=0$, so $f_{1}$ is the trivial $\Sigma_{g}$-bundle [20, 23]. Thus $(f, S)$ is fiber sum indecomposable.

Remark 8. If the section $S$ has self-intersection $2 h-3$, that is, one less than the maximum possible self-intersection, then the first part of the above proof still works and shows that the pair $(f, S)$ cannot be decomposed as a fiber sum of two Lefschetz fibrations over surfaces. However, we shall note that this does not exclude the possibility of the fibration $f$ decomposing as a nontrivial fiber sum; our observation here points out when a fibration and a section are not obtained from fiber summing nontrivial fibrations while pacing their sections.

Lastly, the following proposition will be handy when addressing the main questions of our paper in the case of fiber genus $g=1$ :

Proposition 9. Let $\Sigma_{1}^{1}$ be a torus with one boundary component $\delta$ and let $n$ be an integer. In the mapping class group $\Gamma_{1}^{1}$ of $\Sigma_{1}^{1}$,

- if $n<0$, then $t_{\delta}^{n}$ cannot be written as a product of commutators and right nonseparating Dehn twists,

- if $n \geq 0$ and if $t_{\delta}^{n}$ is a product of commutators and $m$ right nonseparating Dehn twists, then $m=12 n$.

Proof. The mapping class group $\Gamma_{1}^{1}$ is isomorphic to the braid group on three strands and is generated by $t_{a}, t_{b}$ for any two nonseparating simple closed curves intersecting at one point. The first integral homology group $H_{1}\left(\Gamma_{1}^{1}\right)$ is isomorphic to $\mathbb{Z}$ (cf. [10] Theorem 5.1). Let us identify $H_{1}\left(\Gamma_{1}^{1}\right)$ with $\mathbb{Z}$ so that the Dehn twist about a nonseparating curve represents $1 \in \mathbb{Z}$.

Note that we have the equality $t_{\delta}=\left(t_{a} t_{b}\right)^{6}$ so that $t_{\delta}$ represents 12 in $H_{1}\left(\Gamma_{1}^{1}\right) \cong$ $\mathbb{Z}$. It follows that if $t_{\delta}^{n}$ is written as a product of $h$ commutators and $m$ right nonseparating Dehn twists, then $12 n=m$. The proposition follows from this. 


\section{Commutator lengths and surface Bundles WITH MAXIMAL SECTIONS}

If $x$ and $y$ are elements of a group $G$, then we write $[x, y]=x y x^{-1} y^{-1}$ and call it a commutator. The purpose of this section is to write the powers of the Dehn twist about a boundary component on a surface as a product of the least possible number of commutators. More precisely, we will compute the commutator length and the stable commutator length of such a Dehn twist. We then apply it to surface bundles in this section and to Lefschetz fibrations in the next section.

The following lemma will be useful for us. The case $k=1$ was used in 13 .

Lemma 10. Let $\Sigma$ be a compact connected oriented surface. Let a,b, $c, d$ be simple closed curves on $S$ such that there exists a diffeomorphism $f$ mapping $(a, b)$ to $(d, c)$. Then $t_{a}^{k} t_{b}^{-k} t_{c}^{k} t_{d}^{-k}$ is a commutator.

Proof. $t_{a}^{k} t_{b}^{-k} t_{c}^{k} t_{d}^{-k}=t_{a}^{k} t_{b}^{-k} t_{f(b)}^{k} t_{f(a)}^{-k}=t_{a}^{k} t_{b}^{-k} f\left(t_{b}^{k} t_{a}^{-k}\right) f^{-1}=\left[t_{a}^{k} t_{b}^{-k}, f\right]$.

\subsection{Commutator length of the Dehn twist about a boundary component.}

Lantern relation and its generalization. Let $D^{\prime}$ be a disk with boundary $\delta^{\prime}$ from which the interior of three disjoint disks $D_{i}^{\prime}$ are removed. Let $a_{1}^{\prime}, a_{2}^{\prime}, a_{3}^{\prime}$ be the resulting boundary components, i.e., $a_{i}^{\prime}=\partial D_{i}^{\prime}$. Consider the simple closed curves $x_{1}^{\prime}, x_{2}^{\prime}, x_{3}^{\prime}$ on $D^{\prime}$ shown in Figure 1(i). Then we have the lantern relation

$$
t_{\delta^{\prime}} t_{a_{1}^{\prime}} t_{a_{2}^{\prime}} t_{a_{3}^{\prime}}=t_{x_{1}^{\prime}} t_{x_{2}^{\prime}} t_{x_{3}^{\prime}} .
$$

We note that by the Dehn twist about a boundary component we mean the Dehn twist about a simple closed curve parallel to that boundary component.

Suppose now that $D$ is a disk with boundary $\delta$ from which the interior of four disjoint disks $D_{i}$ are removed. Let $a_{1}, a_{2}, a_{3}, a_{4}$ be the resulting boundary components, i.e., $a_{i}=\partial D_{i}$. Consider the simple closed curves $x_{1}, x_{2}, x_{3}, x_{4}, y_{1}, y_{2}$ on $D$ shown in Figure 1(ii).

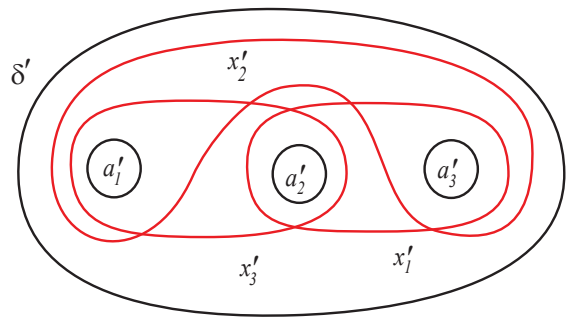

(i)

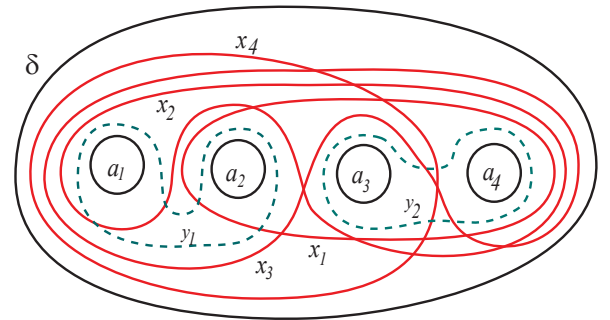

(ii)

FiguRE 1. The curves of the lantern relation and the generalized lantern relation.

Proposition 11. Referring to the simple closed curves in Figure 1(ii), the following generalization of the lantern relation holds in the mapping class group of the fourholed disk D:

$$
t_{\delta}^{2} t_{a_{1}} t_{a_{2}} t_{a_{3}} t_{a_{4}}=t_{x_{1}} t_{x_{2}} t_{x_{3}} t_{x_{4}}
$$


Proof. Note that the simple closed curves $\delta, a_{1}, a_{2}$ and $y_{2}$ bound a sphere with four holes. By the lantern relation we have

$$
t_{\delta} t_{a_{1}} t_{a_{2}} t_{y_{2}}=t_{y_{1}} t_{x_{1}} t_{x_{2}} .
$$

Note also that $\delta, y_{1}, a_{3}$ and $a_{4}$ also bound a sphere with four holes. Again by the lantern relation we have

$$
t_{\delta} t_{y_{1}} t_{a_{3}} t_{a_{4}}=t_{x_{3}} t_{x_{4}} t_{y_{2}} .
$$

From the relations (4) and (5), we get

$$
t_{\delta}^{2} t_{a_{1}} t_{a_{2}} t_{a_{3}} t_{a_{4}} t_{y_{1}} t_{y_{2}}=t_{y_{1}} t_{x_{1}} t_{x_{2}} t_{x_{3}} t_{x_{4}} t_{y_{2}} .
$$

By canceling $t_{y_{1}}$ and $t_{y_{2}}$ from equation (6) we obtain the desired result, the equality (3).

Remark 12 (Generalized lantern relation). The above proof can be generalized in a straightforward fashion to obtain the relation

$$
t_{\delta}^{n-2} t_{a_{1}} t_{a_{2}} \cdots t_{a_{n}}=t_{x_{1}} t_{x_{2}} \cdots t_{x_{n}}
$$

on a disk with $n$ boundary components 1

We are now ready to prove the following theorem:

Theorem 13. Let $g \geq 2, n \geq 1$ and let $\Sigma$ be a compact connected oriented surface of genus $g$ with boundary. Let $\delta$ be one of the boundary components of $\Sigma$. Then the $n^{\text {th }}$ power $t_{\delta}^{n}$ of the Dehn twist about $\delta$ is a product of $\lfloor(n+3) / 2\rfloor$ commutators, where $\lfloor(n+3) / 2\rfloor$ is the largest integer not greater than $(n+3) / 2$.

Proof. First, we rewrite (3) as

$$
t_{\delta}^{2} t_{a_{1}} t_{a_{2}} t_{a_{3}} t_{a_{4}} t_{x_{4}}^{-1}=t_{x_{1}} t_{x_{2}} t_{x_{3}} .
$$

The Dehn twists on the left-hand side of the above equality commute with each other. So for each positive integer $k$, we obtain

$$
\begin{aligned}
t_{\delta}^{2 k} t_{a_{1}}^{k} t_{a_{2}}^{k} t_{a_{3}}^{k} t_{a_{4}}^{k} t_{x_{4}}^{-k} & =\left(t_{x_{1}} t_{x_{2}} t_{x_{3}}\right)^{k} \\
& =\left(t_{x_{1}} t_{x_{2}}\right)\left(t_{x_{1}} t_{x_{2}}\right)^{t_{x_{3}}}\left(t_{x_{1}} t_{x_{2}}\right)^{t_{x_{3}}^{2}} \cdots\left(t_{x_{1}} t_{x_{2}}\right)^{t_{x_{3}}^{k-1}} t_{x_{3}}^{k} \\
& =\left(\prod_{i=1}^{k}\left(t_{x_{1}} t_{x_{2}}\right)^{t_{x_{3}}^{i-1}}\right) t_{x_{3}}^{k}
\end{aligned}
$$

or

$$
t_{\delta}^{2 k} t_{a_{1}}^{k} t_{a_{2}}^{k} t_{a_{3}}^{k} t_{a_{4}}^{k}=\left(\prod_{i=1}^{k}\left(t_{x_{1}} t_{x_{2}}\right)^{t_{x_{3}}^{i-1}}\right) t_{x_{3}}^{k} t_{x_{4}}^{k} .
$$

Here, $t_{a}^{f}$ denotes the conjugation $f t_{a} f^{-1}$. By multiplying both sides of (7) by $t_{a_{1}}^{-k} t_{a_{2}}^{-k} t_{a_{3}}^{-k} t_{a_{4}}^{-k}$, we get

$$
t_{\delta}^{2 k}=\left(\prod_{i=1}^{k}\left(t_{x_{1}} t_{a_{2}}^{-1} t_{x_{2}} t_{a_{1}}^{-1}\right)^{t_{x_{3}}^{i-1}}\right)\left(t_{x_{3}}^{k} t_{a_{4}}^{-k} t_{x_{4}}^{k} t_{a_{3}}^{-k}\right) .
$$

Consider the four-holed disk $D$ in Figure 1(ii) embedded in the surface $\Sigma$ of genus $g \geq 2$ as in Figure 2(i), so that $a_{1}=a_{4}$.

\footnotetext{
${ }^{1}$ As Burak Ozbagci kindly pointed out to us, this generalized lantern relation is equivalent to a relation obtained by Plamenevskaya and Van Horn-Morris in [18.
} 

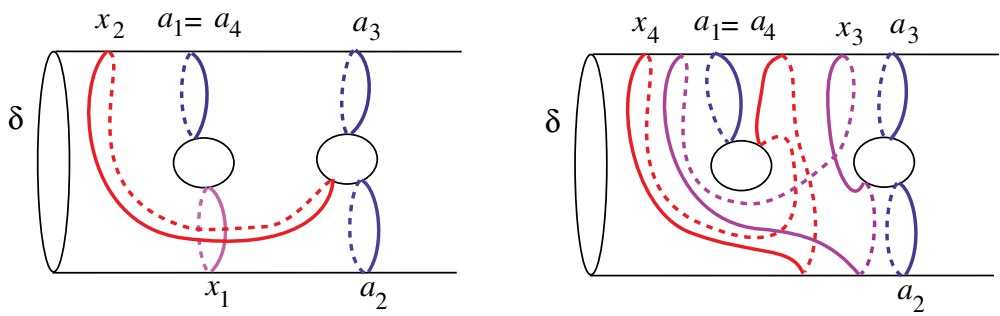

(i)

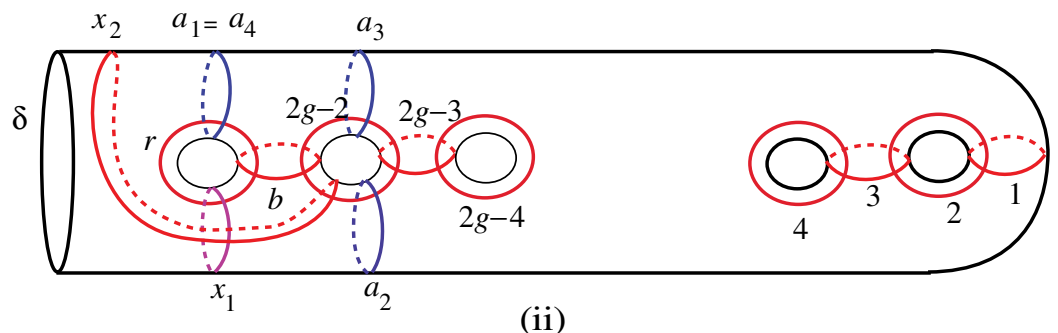

(ii)

Figure 2. The curve labeled $i$ is $c_{i}$ for $i=1,2, \ldots, 2 g-2$.

Suppose first that $n=2 k$. It follows from Lemma 10 that $t_{x_{3}}^{k} t_{a_{4}}^{-k} t_{x_{4}}^{k} t_{a_{3}}^{-k}$ is a commutator, and so is $t_{x_{1}} t_{a_{2}}^{-1} t_{x_{2}} t_{a_{1}}^{-1}$. Since a conjugate of a commutator is again a commutator, we have expressed $t_{\delta}^{n}$ as a product of $k+1$ commutators, proving the theorem for even $n$.

Suppose now that $n=2 k+1$. We rewrite the lantern relation (5) as

$$
t_{x_{3}}^{-1} t_{\delta}=t_{x_{4}} t_{a_{3}}^{-1} t_{a_{4}}^{-1} t_{y_{2}} t_{y_{1}}^{-1} \text {. }
$$

From (8) and (9), we get

$$
t_{x_{3}}^{-1} t_{\delta}^{2 k+1}=\left(\prod_{i=1}^{k}\left(t_{x_{1}} t_{a_{2}}^{-1} t_{x_{2}} t_{a_{1}}^{-1}\right)^{t_{x_{3}}^{i-1}}\right)\left(t_{x_{3}}^{k} t_{a_{4}}^{-k-1} t_{x_{4}}^{k+1} t_{a_{3}}^{-k-1}\right)\left(t_{y_{2}} t_{y_{1}}^{-1}\right)
$$

or

$$
t_{\delta}^{2 k+1}=\left(\prod_{i=1}^{k}\left(t_{x_{1}} t_{a_{2}}^{-1} t_{x_{2}} t_{a_{1}}^{-1}\right)^{t_{x_{3}}^{i}}\right)\left(t_{x_{3}}^{k+1} t_{a_{4}}^{-k-1} t_{x_{4}}^{k+1} t_{a_{3}}^{-k-1}\right)\left(t_{y_{2}} t_{y_{1}}^{-1}\right) .
$$

It follows again from Lemma 10 that each of the elements $t_{x_{1}} t_{a_{2}}^{-1} t_{x_{2}} t_{a_{1}}^{-1}$ and $t_{x_{3}}^{k+1} t_{a_{4}}^{-k-1} t_{x_{4}}^{k+1} t_{a_{3}}^{-k-1}$ is a commutator. Since $t_{y_{2}} t_{y_{1}}^{-1}$ is also a commutator, it follows that $t_{\delta}^{2 k+1}$ is a product of $k+2$ commutators.

This proves the theorem.

Corollary 14. Let $g \geq 2, h \geq 1$, and let $\Sigma$ be a compact connected oriented surface of genus $g$ with boundary. Let $\delta$ be one of the boundary components of $\Sigma$. Then for each $2-2 h \leq k \leq 2 h-2$, $t_{\delta}^{k}$ is a product of $h$ commutators.

3.2. Stable commutator lengths in mapping class groups of surfaces with boundary. Let $G$ be a group and let $[G, G]$ denote the commutator subgroup of $G$, the subgroup generated by the set of all commutators $[x, y]=x y x^{-1} y^{-1}$ for 
$x, y \in G$. Let $x$ be an element in $[G, G]$ so that it can be written as a product of commutators. The commutator length $\operatorname{cl}(x)$ of $x$ in $G$ is defined as the minimal number of commutators needed to express $x$ as a product of commutators. The stable commutator length of $x$ is the limit

$$
\operatorname{scl}(x)=\lim _{n \rightarrow \infty} \frac{\operatorname{cl}\left(x^{n}\right)}{n} .
$$

This limit exists since the function $\mathrm{cl}$ is subadditive: $\operatorname{cl}\left(x^{n+m}\right) \leq \operatorname{cl}\left(x^{n}\right)+\operatorname{cl}\left(x^{m}\right)$. Note also that $\operatorname{cl}\left(y x y^{-1}\right)=\operatorname{cl}(x), \operatorname{cl}\left(x^{-1}\right)=\operatorname{cl}(x)$ and $\operatorname{scl}\left(x^{k}\right)=|k| \operatorname{scl}(x)$ for any $x \in[G, G]$ and $y \in G$. For more on the functions $\mathrm{cl}$ and scl, we refer to $[6]$.

Theorem 15. Let $g \geq 2$ and let $\Sigma$ be a compact connected oriented surface of genus $g$ with at least one boundary component. Let $\delta$ be one of the boundary components of $\Sigma$. Then for any nonzero integer $n$ the commutator length of $t_{\delta}^{n}$ is

$$
\operatorname{cl}\left(t_{\delta}^{n}\right)=\lfloor(|n|+3) / 2\rfloor .
$$

In particular, we have $\operatorname{scl}\left(t_{\delta}\right)=1 / 2$.

Proof. Since $\operatorname{cl}\left(t_{\delta}^{-n}\right)=\operatorname{cl}\left(t_{\delta}^{n}\right)$ we may assume that $n$ is positive. By Theorem 13, $\operatorname{cl}\left(t_{\delta}^{n}\right) \leq\lfloor(n+3) / 2\rfloor$.

Suppose, on the other hand, that $c\left(t_{\delta}^{n}\right)=h$. Writing $t_{\delta}^{n}$ as a product of $h$ commutators gives us a genus- $g$ surface bundle over a surface of genus $h$ with a section of self-intersection $-n$. By Proposition 4, we get $n \leq 2 h-2$. Hence, we have $c\left(t_{\delta}^{n}\right) \geq(n+2) / 2$. The theorem follows from this.

Theorem 15 computes the first precise nonzero value of the stable commutator length of an element in the mapping class group of a surface. In earlier works bounds on stable commutator lengths of elements of mapping class groups were established without such an explicit calculation [7, 11.

Remark 16 (An alternative proof of $\operatorname{scl}\left(t_{\delta}\right) \leq \frac{1}{2}$ ). The inequality $\operatorname{scl}\left(t_{\delta}\right) \leq \frac{1}{2}$ may alternatively be obtained by using quasimorphisms: Given a group $G$, a function $\phi: G \rightarrow \mathbb{R}$ is called a quasimorphism if there exists a least constant $D_{\phi} \geq 0$, called the defect of $\phi$, such that $|\phi(x y)-\phi(x)-\phi(y)| \leq D_{\phi}$ for all $x, y \in G$. A quasimorphism $\phi$ is called homogeneous if it satisfies the additional property that $\phi\left(x^{n}\right)=n \phi(x)$ for all $x \in G$ and integers $n$. For more on quasimorphisms, we refer to [6].

We recall the basic properties of homogeneous quasimorphisms we use. If $\phi$ is a homogeneous quasimorphism, then $\phi$ is constant on conjugacy classes. Therefore, a homogeneous quasimorphism on the mapping class group takes the same value on Dehn twists about nonseparating simple closed curves. For any two elements $x, y$ in $G$, if $x y=y x$, then

$$
\begin{aligned}
|\phi(x y)-\phi(x)-\phi(y)| & =\lim _{n \rightarrow \infty}|\phi(x y)-\phi(x)-\phi(y)| \\
& =\lim _{n \rightarrow \infty} \frac{1}{n}\left|\phi\left((x y)^{n}\right)-\phi\left(x^{n}\right)-\phi\left(y^{n}\right)\right| \\
& =\lim _{n \rightarrow \infty} \frac{1}{n}\left|\phi\left(x^{n} y^{n}\right)-\phi\left(x^{n}\right)-\phi\left(y^{n}\right)\right| \\
& \leq \lim _{n \rightarrow \infty} \frac{1}{n} D_{\phi} \\
& =0 .
\end{aligned}
$$

Hence $\phi(x y)=\phi(x)+\phi(y)$. 
Let $Q$ be the set of homogeneous quasimorphisms on $G$. Bavard's main theorem in [2] states that for any $x \in[G, G]$,

$$
\operatorname{scl}(x)=\sup _{\phi \in Q} \frac{|\phi(x)|}{2 D_{\phi}} .
$$

Now, the proof proceeds as follows: Let $\phi$ be a homogeneous quasimorphism on the mapping class group of $\Sigma$, which is a surface of genus $g \geq 2$. Let $\delta$ be a boundary component. We may choose six nonseparating curves $a_{1}, a_{2}, a_{3}, x_{1}, x_{2}, x_{3}$ on $\Sigma$ such that we have the lantern relation $t_{\delta} t_{a_{1}} t_{a_{2}} t_{a_{3}}=t_{x_{1}} t_{x_{2}} t_{x_{3}}$. We then have

$$
\begin{aligned}
\phi\left(t_{x_{1}} t_{x_{2}}\right) & =\phi\left(t_{\delta} t_{a_{1}} t_{a_{2}} t_{a_{3}} t_{x_{3}}^{-1}\right) \\
& =\phi\left(t_{\delta}\right)+\phi\left(t_{a_{1}}\right)+\phi\left(t_{a_{2}}\right)+\phi\left(t_{a_{3}}\right)-\phi\left(t_{x_{3}}\right) \\
& =\phi\left(t_{\delta}\right)+2 \phi\left(t_{a_{1}}\right) .
\end{aligned}
$$

From the definition of a quasimorphism and the properties of $\phi$,

$$
\begin{aligned}
D_{\phi} & \geq\left|\phi\left(t_{x_{1}} t_{x_{2}}\right)-\phi\left(t_{x_{1}}\right)-\phi\left(t_{x_{2}}\right)\right| \\
& =\left|\phi\left(t_{\delta}\right)+2 \phi\left(t_{a_{1}}\right)-\phi\left(t_{x_{1}}\right)-\phi\left(t_{x_{2}}\right)\right| \\
& =\left|\phi\left(t_{\delta}\right)\right| .
\end{aligned}
$$

Thus, by Bavard's result (11), we have $\operatorname{scl}\left(t_{\delta}\right) \leq \frac{1}{2}$.

Remark 17. The last part of our theorem on the stable commutator length $\operatorname{scl}\left(t_{\delta}\right)=\frac{1}{2}$ can be compared to the calculation of the "vertical euler class" $\|e\|=\frac{1}{2}$ by Morita in [17.

Lastly, we look at the relevant stable mapping class group. Let $\Sigma_{g}^{k}$ denote the surface of genus $g$ with $k \geq 2$ boundary components. Let us distinguish a boundary component $\partial$. By attaching a torus $T$ with two boundary components to $\Sigma_{g}^{k}$ along $\partial$, we obtain a surface $\Sigma_{g+1}^{k}$ of genus $g+1$ with a distinguished boundary component, $\partial$, the common boundary component of $T$ and $\Sigma_{g+1}^{k}$, and an injection $\Gamma_{g}^{k} \rightarrow \Gamma_{g+1}^{k}$. By taking the direct limit we get a group

$$
\Gamma_{\infty}^{k-1}=\lim _{g \rightarrow \infty} \Gamma_{g}^{k}
$$

The group $\Gamma_{\infty}^{k-1}$ is the group of isotopy classes of compactly supported diffeomorphisms of a one-ended countable infinite genus surface with $k-1$ boundary components.

If $\delta$ is a boundary component of $\Sigma_{g}^{k}$ other than $\partial$, then the Dehn twist $t_{\delta}$ may be seen as an element of $\Gamma_{\infty}^{k-1}$. Theorem 15 implies that the stable commutator length of $t_{\delta}$ in $\Gamma_{\infty}^{k-1}$ is $\frac{1}{2}$. The same conclusion holds if one stabilizes the surface along possible different boundary components but not along $\delta$. (In this case the stable mapping class group depends on the stabilization.) In particular, $\operatorname{scl}\left(t_{\delta}\right)$ is stable. This may be compared with Harer's homology stability [9] and contrasts a result of Kotschick [14] who showed that the function scl vanishes on $\Gamma_{\infty}=\Gamma_{\infty}^{0}$.

3.3. Surface bundles with maximal sections. We note that in any group $G$, if $a_{i}$ commutes with $b_{j}$, then $\left[a_{1}, a_{2}\right]\left[b_{1}, b_{2}\right]=\left[a_{1} b_{1}, a_{2} b_{2}\right]$. This fact will be used repeatedly below.

Theorem 18. Let $h \geq 1$. For every $g \geq 2$ and $2-2 h \leq k \leq 2 h-2$, there exists a $\Sigma_{g}$-bundle over $\Sigma_{h}$, admitting a section of self-intersection $k$ and, in particular, 
a maximal section. Moreover, provided $g \geq 8 h-8$, there exists a $\Sigma_{g}$-bundle over $\Sigma_{h}$, admitting $4 h-3$ disjoint sections $S_{i}$ with $\left[S_{i}\right]^{2}=i$, for $2-2 h \leq i \leq 2 h-2$.

Proof. Let $\Sigma$ be a compact connected oriented surface of genus $g$ with one boundary component $\delta$. By Theorem 13, we may express $t_{\delta}^{2-2 h}$ as a product of $h$ commutators in $\Gamma_{g}^{1}$. Capping off the boundary component $\delta$ of $\Sigma$ gives us a relation in $\Gamma_{g}$ which is the monodromy representation of a genus $-g$ surface bundle over a genus- $h$ surface with a section, and the self-intersection number of this section is $2 h-2$. This proves the first assertion of the theorem.

For the second part of the theorem, let $Z$ be a compact connected oriented surface of genus $l \geq 0$ with $4 h-3$ boundary components, $d_{i}, 2-2 h \leq i \leq 2 h-2$. For each $2-2 h \leq i \leq 2 h-2$ with $i \neq 0$, let $Z_{i}$ be a compact connected orientable surface of genus two with two boundary components $d_{i}^{\prime}$ and $\delta_{i}$. Glue each $Z_{i}$ to $Z$ by identifying $d_{i}$ and $d_{i}^{\prime}$ to obtain a connected oriented surface $\Sigma$ of genus $g=8 h-8+l$ with $4 h-3$ boundary components $\delta_{i}$, where $\delta_{0}=d_{0}$.

By Corollary 14, there are diffeomorphisms $f_{i 1}, g_{i 1}, f_{i 2}, g_{i 2}, \ldots, f_{i h}, g_{i h}$ of $\Sigma$ supported on the subsurface $Z_{i}$ such that

$$
t_{\delta_{i}}^{i}=\left[f_{i 1}, g_{i 1}\right]\left[f_{i 2}, g_{i 2}\right] \cdots\left[f_{i h}, g_{i h}\right] .
$$

Note that if $i \neq j$, then $f_{i s}$ and $g_{i s}$ commute with $f_{j t}$ and $g_{j t}$. It now follows that

$$
\prod_{i=2-2 h}^{2 h-2} t_{\delta_{i}}^{i}
$$

is a product of $h$ commutators. Hence, we obtained a relation yielding a monodromy representation for a surface bundle with the desired properties.

Remark 19. The second construction in our proof of Theorem 18 can be derived from the first one geometrically. To do so, we first take the separate $\Sigma_{g_{i}}$-bundle $f_{i}$ over $\Sigma_{h}$ with a section $S_{i}$ of self-intersection $\left[S_{i}\right]^{2}=i$ for each integer $i$ in the interval $[2-2 h, 2 h-2]$, for any chosen collection of $g_{i} \geq 2$ satisfying $\sum_{i} g_{i}=g \geq 8 h-8$. Then we observe that the relations handed us by Corollary 14 hold on surfaces with more than one boundary component as well. Thus we can get self-intersection zero sections of each $f_{i}$ disjoint from $S_{i}$. Hence, we can section sum the $f_{i}$ along these self-intersection zero sections to obtain a $\Sigma_{g}$-bundle over $\Sigma_{h}$, provided $g \geq 8 h-8$.

We now look at the remaining cases for the fiber and the base genera not covered by Theorem 18 .

If $g=0, h \geq 0$, then the total space of the bundle is an $S^{2}$-bundle over $\Sigma_{h}$, which is classified by the Euler number $k$ and the base genus $h$. Each bundle corresponding to Euler number $k$ admits a section of self-intersection $k$. When $g=1$, we swing to another extreme situation: By Proposition 9] a section of a $T^{2}$-bundle over any $\Sigma_{h}$ can only attain a zero self-intersection number.

When the base genus $h=0$, there is a unique $\Sigma_{g}$-bundle over $S^{2}$ for $g>$ 1, namely the trivial bundle $\Sigma_{g} \times S^{2}$ given by the projection onto the second component. There are many $T^{2}$-bundles over $S^{2}$, however only one of them admits a section: the trivial bundle on $T^{2} \times S^{2}$. (See for instance [4] (Lemma 10).) So the self-intersection number of a section of a $\Sigma_{g}$-bundle over $S^{2}$ for $g \geq 1$ is always zero. 
These observations in the remaining cases can be summarized as:

Proposition 20. For any $k \in \mathbb{Z}$ and $h \geq 0$, there exists an $S^{2}$-bundle over $\Sigma_{h}$ admitting a section $S$ with $[S]^{2}=k$. For any $g \geq 1$, the only $\Sigma_{g}$-bundle over $S^{2}$ admitting a section $S$ is the trivial one, for which $[S]^{2}$ is necessarily zero. For any $h \geq 0$, any section $S$ of a $T^{2}$-bundle over $\Sigma_{h}$ has $[S]^{2}=0$.

\section{LEFSCHETZ FibRATIONS WITH AN ARBITRARILY LARGE NUMBER OF CRITICAL POINTS}

In this section, we focus on the following problem: For fixed nonnegative integers $g$ and $h$, is there an upper bound on the number of critical points of a relatively minimal genus-g Lefschetz fibration over a genus- $h$ surface, admitting a maximal section?

One can easily inflate the number of critical points of Lefschetz fibrations with fixed fiber and base genera by introducing reducible fibers or by performing fiber sums with the same genus fibrations over the 2-sphere. Our assumptions on relative minimality of the fibrations and on the existence of maximal sections (see Proposition 7) are therefore essential. On the other hand, it was shown by Ivan Smith [20] and independently by Andras Stipsicz 23] that the self-intersection of a section of a nontrivial Lefschetz fibration over the 2 -sphere can be at most -1 , which is achieved by many Lefschetz fibrations, and most importantly by those that are obtained from Lefschetz pencils. So the case $h=0$ of our question is the one in 1 .

The problem is vacuous for $g=0$ because of our natural assumption on the minimal relativity. For $g=1$, and for any fixed $h \geq 0$, Proposition 9 implies that the number of critical points $m$ of a Lefschetz fibration is determined by the selfintersection number $n$ of a section by the equation $m=-12 n$. In particular, every section should have the same self-intersection number. So the number of critical points is bounded in this case. As shown by Ivan Smith [21] (also see 19]), the number of critical points is also bounded when $h=0$ and $g=2$. Our main theorem in this section shows that this fails to be true when the base genus $h$ is positive:

We will need the following well-known lemma in the proof of our main theorem of this section:

Lemma 21. Let $c_{1}, c_{2}, \ldots, c_{2 l+1}$ be a sequence of simple closed curves on an oriented surface such that $c_{i}$ and $c_{j}$ are disjoint if $|i-j| \geq 2$ and that $c_{i}$ and $c_{i+1}$ intersect at one point. A regular neighborhood of $c_{1} \cup c_{2} \cup \cdots \cup c_{2 l+1}$ is a subsurface of genus $l$ with two boundary components, say $d_{1}$ and $d_{2}$. We then have the chain relation

$$
\left(t_{c_{1}} t_{c_{2}} \cdots t_{c_{2 l+1}}\right)^{2 l+2}=t_{d_{1}} t_{d_{2}} .
$$

Theorem 22. Let $g \geq 2$ and $h \geq 1$ be fixed integers. For any positive integer $M$, there exists a relatively minimal genus-g Lefschetz fibration over a surface of genus $h$ admitting a section of maximal self-intersection such that the number of critical points is greater than $M$.

Proof. By Proposition 4, the self-intersection of a maximal section of a genus $-g$ Lefschetz fibration over a surface of genus $h \geq 1$ is $2 h-2$. Therefore, it suffices to write $t_{\delta}^{2-2 h}$ as a product of $h$ commutators and a number of right Dehn twists such that the number of right Dehn twists is at least $M$. 
Suppose first that $h \geq 2$. Consider the surface $\Sigma_{g}^{1}$ in Figure 2(ii) and the curves on it. By (8), we have the following equality the mapping class group of $\Sigma_{g}^{1}$ :

$$
t_{\delta}^{-2 k}=\left(t_{a_{3}}^{k} t_{x_{4}}^{-k} t_{a_{4}}^{k} t_{x_{3}}^{-k}\right)\left(\prod_{i=1}^{k}\left(t_{a_{1}} t_{x_{2}}^{-1} t_{a_{2}} t_{x_{1}}^{-1}\right)^{t_{x_{3}}^{k-i}}\right) .
$$

By writing $k=h-1, C_{h-1}=t_{a_{3}}^{h-1} t_{x_{4}}^{1-h} t_{a_{4}}^{h-1} t_{x_{3}}^{1-h}, C=t_{a_{1}} t_{x_{2}}^{-1} t_{a_{2}} t_{x_{1}}^{-1}$ and $C_{i}=$ $t_{x_{3}}^{i} C t_{x_{3}}^{-i}, 1 \leq i \leq h-2$, we may rewrite (12) as

$$
t_{\delta}^{2-2 h}=C_{h-1} C_{h-2} \cdots C_{2} C_{1} C,
$$

where each $C_{i}$ is a commutator.

On the other hand, by Lemma 21, we have the relation $\left(t_{a_{1}} t_{r} t_{b}\right)^{4}=t_{x_{2}} t_{a_{3}}$. From this we easily get

$$
t_{r} t_{a_{1}} t_{b} t_{r}\left(t_{a_{1}} t_{r} t_{b}\right)^{2}=t_{x_{2}} t_{a_{3}} t_{a_{1}}^{-2}
$$

Let $T_{1}$ denote the left-hand side of the equality (14) so that $T_{1}$ is a product of 10 right Dehn twists. Thus, for any integer $m$, we have

$$
T_{1}^{m}=t_{x_{2}}^{m} t_{a_{3}}^{m} t_{a_{1}}^{-2 m}
$$

or

$$
T_{1}^{m} t_{a_{1}}^{2 m} t_{a_{3}}^{-m} t_{x_{2}}^{-m}=1 .
$$

By Lemma 21, we also have the relation

$$
\left(t_{c_{1}} t_{c_{2}} \cdots t_{c_{2 g-3}} t_{c_{2 g-2}} t_{b}\right)^{2 g}=t_{x_{1}} t_{a_{1}} .
$$

The left-hand side of this equality may be written as $T_{2}\left(t_{c_{1}} t_{c_{2}} \cdots t_{c_{2 g-3}}\right)^{2 g-2}$, where $T_{2}$ is a product of right Dehn twists. (The number of Dehn twists in $T_{2}$ is $8 g-6$.) Applying Lemma 21 once again, we see that it is also equal to $T_{2} t_{a_{2}} t_{a_{3}}$. Hence, we obtain from (16) that

$$
T_{2}^{m}=t_{x_{1}}^{m} t_{a_{1}}^{m} t_{a_{2}}^{-m} t_{a_{3}}^{-m}
$$

or

$$
t_{x_{1}}^{-m} t_{a_{1}}^{-m} t_{a_{2}}^{m} t_{a_{3}}^{m} T_{2}^{m}=1
$$

Finally, by using (15) and (17), we write $C$ as

$$
\begin{aligned}
C & =t_{a_{1}} t_{x_{2}}^{-1} t_{a_{2}} t_{x_{1}}^{-1} \\
& =t_{x_{2}}^{-1} t_{x_{1}}^{-1} t_{a_{1}} t_{a_{2}} \\
& =\left(T_{1}^{m} t_{a_{1}}^{2 m} t_{a_{3}}^{-m} t_{x_{2}}^{-m}\right) t_{x_{2}}^{-1} t_{x_{1}}^{-1} t_{a_{1}} t_{a_{2}}\left(t_{x_{1}}^{-m} t_{a_{1}}^{-m} t_{a_{2}}^{m} t_{a_{3}}^{m} T_{2}^{m}\right) \\
& =T_{1}^{m} t_{x_{2}}^{-m-1} t_{x_{1}}^{-m-1} t_{a_{1}}^{m+1} t_{a_{2}}^{m+1} T_{2}^{m} \\
& =\left(T_{1}^{m} t_{x_{2}}^{-m-1} t_{x_{1}}^{-m-1} t_{a_{1}}^{m+1} t_{a_{2}}^{m+1} T_{1}^{-m}\right) T_{1}^{m} T_{2}^{m} .
\end{aligned}
$$

If we let $C_{0}^{(m)}=T_{1}^{m}\left(t_{x_{1}}^{-m-1} t_{x_{2}}^{-m-1} t_{a_{1}}^{m+1} t_{a_{2}}^{m+1}\right) T_{1}^{-m}$, then $C_{0}^{(m)}$ is a commutator. By (13), we then have

$$
t_{\delta}^{2-2 h}=C_{h-1} C_{h-2} \cdots C_{2} C_{1} C_{0}^{(m)} T_{1}^{m} T_{2}^{m},
$$

so that $t_{\delta}^{2-2 h}$ is expressed as a product of $h$ commutators and $4(2 g+1) m$ right Dehn twists. Taking $m$ large enough completes the proof of the theorem in the case $h \geq 2$. 
Suppose now that $h=1$ so that $2 h-2=0$. Consider the surface $\Sigma_{g}^{1}$ and the curves on it given in Figure 2(ii) once again. Let $2 \leq l \leq g$ and let $d_{1}$ and $d_{2}$ be the boundary components of a regular neighborhood of $c_{1} \cup c_{2} \cup c_{3} \cup \cdots \cup c_{2 l-1}$, where $c_{2 g-1}=b$ and $c_{2 g}=r$.

By Lemma 21, we have the relation $\left(t_{c_{1}} t_{c_{2}} \cdots t_{c_{2 l-1}}\right)^{2 l}=t_{d_{l}} t_{d_{2}}$. From this we get

$$
\left(t_{c_{2}} \cdots t_{c_{2 l-1}}\right)\left(t_{c_{1}} t_{c_{2}} \cdots t_{c_{2 l-1}}\right)^{2 l-2}\left(t_{c_{1}} t_{c_{2}} \cdots t_{c_{2 l-2}}\right)=t_{d_{l}} t_{d_{2}} t_{c_{1}}^{-1} t_{c_{2 l-1}}^{-1} .
$$

Let $T(l)$ denote the left-hand side of (18) so that $T(l)$ is a product of right Dehn twists. Thus, for any integer $m$ we have

$$
(T(l))^{m}=t_{d_{l}}^{m} t_{d_{2}}^{m} t_{c_{1}}^{-m} t_{c_{2 l-1}}^{-m}
$$

or

$$
t_{\delta}^{0}=1=t_{d_{l}}^{-m} t_{d_{2}}^{-m} t_{c_{1}}^{m} t_{c_{2 l-1}}^{m}(T(l))^{m} .
$$

If we let $C(l)=t_{d_{l}}^{-m} t_{d_{2}}^{-m} t_{c_{1}}^{m} t_{c_{2 l-1}}^{m}$, then $C(l)$ is a commutator. By (19), we then have

$$
t_{\delta}^{0}=1=C(l)(T(l))^{m} .
$$

Again, taking $m$ large enough completes the proof in the case $h=1$.

Remark 23. It should be evident that the techniques used in the proof of Theorem 22 fall short in covering the remaining case $h=0$, and thus the question remains open for $h=0$ and $g \geq 3$.

Remark 24. Proposition 7 implies that the Lefschetz fibration and section pairs we construct in Theorem 22 are fiber sum indecomposable. It is a priori unclear whether or not these fibrations (but not the pairs) can be decomposed as fiber sums where one of the fibrations is over the 2-sphere. We shall note, however, by employing slightly different manipulations of mapping class group relations, that we can construct examples as in Theorem 22, meeting these additional conditions as well, relying on Smith's fillability criterion in 20. Nevertheless, we will skip this rather repetitive inclusion here, which does not seem to relate to the core problem in question.

Remark 25. Theorem 22 shows the existence of genus $-g$ Lefschetz fibrations with maximal sections over surfaces of positive genera, similar to our result cast in Theorem 18 ,

\section{ACKNOWLEDGEMENTS}

The first author was partially supported by the NSF grant DMS-0906912. The second author thanks the Max-Planck Institut für Mathematik in Bonn for its generous support and wonderful research environment. The third author thanks Hisaaki Endo, Kenta Hayano, Dieter Kotschick, and Masatoshi Sato for their comments on the content of this paper.

\section{REFERENCES}

[1] Denis Auroux; "Mapping class group factorizations and symplectic 4-manifolds: Some open problems", Problems on mapping class groups and related topics, 123-132, Proc. Sympos. Pure Math., 74, Amer. Math. Soc., Providence, RI, 2006. MR2264537 (2007h:53134)

[2] Christophe Bavard; “Longueur stable des commutateurs", L'Enseignement Mathématique 37 (1991), 109-150. MR1115747 (92g:20051) 
[3] Jonathan Bowden; On closed leaves of foliations, multisections and stable commutator lengths, J. Topol. Anal. 3 (2011), no. 4, 491-509. MR2887673

[4] R. Inanc Baykur and Seiichi Kamada; "Classification of broken Lefschetz fibrations with small fiber genera", preprint; http://arxiv.org/abs/1010.5814.

[5] Volker Braungardt, Dieter Kotschick; "Clustering of critical points in Lefschetz fibrations and the symplectic Szpiro inequality", Trans. Amer. Math. Soc. 355 (2003), no. 8, 3217 3226. MR1974683 (2004b:57034)

[6] Danny Calegari; "Scl", MSJ Memoirs, 20. Mathematical Society of Japan, Tokyo, 2009. MR2527432(2011b:57003)

[7] Hisaaki Endo and Dieter Kotschick; "Bounded cohomology and non-uniform perfection of mapping class groups", Invent. Math. 144 (2001), no. 1, 169-175. MR.1821147(2001m:57046)

[8] Robert E. Gompf and Andras I. Stipsicz; "4-manifolds and Kirby calculus", Graduate Studies in Mathematics, vol. 20, American Math. Society, Providence 1999. MR.1707327 (2000h:57038)

[9] John Harer; "Stability of the homology of the mapping class groups of orientable surfaces", Ann. of Math. (2) 121 (1985), no. 2, 215-249. MR786348(87f:57009)

[10] Mustafa Korkmaz; "Low-dimensional homology groups of mapping class groups: A survey", Turkish J. Math. 26 (2002), no. 1, 101-114. MR1892804 (2003f:57002)

[11] Mustafa Korkmaz; "Stable commutator length of a Dehn twist", Michigan Math. J. 52 (2004), no. 1, 23-31. MR2043394 (2005b:57003)

[12] Mustafa Korkmaz; "Problems on homomorphisms of mapping class groups", Problems on mapping class groups and related topics, 81-89, Proc. Sympos. Pure Math., 74, Amer. Math. Soc., Providence, RI, 2006. MR2264533(2007f:57029)

[13] Mustafa Korkmaz and Burak Ozbagci; "Minimal number of singular fibers in a Lefschetz fibration”, Proc. Amer. Math. Soc. 129 (2000), no. 5, 1545-1549. MR1713513 (2001h:57019)

[14] Dieter Kotschick; "Stable length in stable groups", Groups of diffeomorphisms in honor of Shigeyuki Morita on the occasion of his 60th birthday, Advanced Studies in Pure Mathematics 52, Mathematical Society of Japan 2008, pp. 401-413. MR2509718 (2011j:57003)

[15] Dieter Kotschick; "Quasi-homomorphisms and stable lengths in mapping class groups", Proc. Amer. Math. Soc. 132(2004), no. 11, 3167-3175. MR2073290 (2005e:20065)

[16] Ai-Ko Liu; "Some new applications of general wall crossing formula, Gompf's conjecture and its applications", Math. Res. Lett. 3 (1996), no. 5, 569-585. MR1418572 (97k:57038)

[17] Shigeyuki Morita; "Characteristic classes of surface bundles and bounded cohomology", A fête of topology, 233-257, Academic Press, Boston, MA, 1988. MR0928403 (89c:57030)

[18] Olga Plamenevskaya, Jeremy Van Horn-Morris; "Planar open books, monodromy factorizations and symplectic fillings", Geom. Topol. 14 (2010), no. 4, 2077-2101. MR2740642 (2012c:57047)

[19] Yoshihisa Sato; "2-spheres of square -1 and the geography of genus-2 Lefschetz fibrations", J. Math. Sci. Univ. Tokyo 15 (2008), no. 4, 461-491. MR2546906 (2011c:57059)

[20] Ivan Smith; "Geometric monodromy and the hyperbolic disc", Q. J. Math. 52 (2001), 217228. MR 1838364 (2002c:57046)

[21] Ivan Smith; "Lefschetz pencils and divisors in moduli space", Geometry and Topology 5 (2001), 579-608. MR1833754 (2002f:57056)

[22] Andras Stipsicz; "Chern numbers of certain Lefschetz fibrations", Proc. Amer. Math. Soc. 128 (1999), no. 6, 1845-1851. MR1641113 (2000j:57062)

[23] Andras Stipsicz; "Indecomposability of certain Lefschetz fibrations", Proc. Amer. Math. Soc. 129 (2001), no. 5, 1499-1502. MR.1712877 (2001h:57029)

Max Planck Institut für Mathematik, Bonn, Germany - And - Department of Mathematics, Brandeis University, Waltham, Massachusetts 02453

E-mail address: baykur@mpim-bonn.mpg.de, baykur@brandeis.edu

Department of Mathematics, Middle East Technical University, Ankara, Turkey

E-mail address: korkmaz@metu.edu.tr

Department of Mathematics, Osaka University, Osaka, Japan

E-mail address: n-monden@cr.math.sci.osaka-u.ac.jp 